\title{
Distribuição geográfica de Lutzomyia (Nyssomyia) whitmani (Antunes \& Coutinho, 1939) no Estado de Mato Grosso
}

\author{
Geographical distribution of Lutzomyia (Nyssomyia) whitmani \\ (Antunes \& Coutinho, 1939) in the State of Mato Grosso
}

\author{
Nanci Akemi Missawa ${ }^{1}$, Giovana Belém Moreira Lima Maciel ${ }^{1}$ \\ e Hilda Rodrigues ${ }^{1}$
}

\begin{abstract}
RESUMO
No Estado de Mato Grosso, $100 \%$ dos municípios apresentam registros de casos autóctones de leishmaniose tegumentar americana. 0 presente trabalho objetivou verificar a distribuição geográfica de Lutzomyia whitmani no estado. Mato Grosso possui três ecossistemas distintos, o cerrado, o pantanal e área de domínio amazônico. Os dados sobre a ocorrência de Lutzomyia whitmani foram obtidos a partir de relatórios de pesquisa entomológica realizados pelo Núcleo de Entomologia da Fundação Nacional de Saúde no período de 1996 a 2000 e de 2001 a 2006 pelo Laboratório de Entomologia da Secretaria Estadual de Saúde de Mato Grosso. Foram realizadas pesquisas entomológicas em 83 (59,7\%) dos 139 municípios de Mato Grosso. Lutzomyia whitmani foi capturado em 70 (84,3\%) municípios, com ampla distribuição em todos os tipos de vegetação.
\end{abstract}

Palavras-chaves: Lutzomyia whitmani. Leishmaniose tegumentar americana. Mato Grosso.

\begin{abstract}
In the State of Mato Grosso, $100 \%$ of the municipalities have records of autochthonous cases of American cutaneous leishmaniasis. The present study had the aim of investigating the geographical distribution of the Lutzomyia whitmani in the state. Mato Grosso has three distinct ecosystems: the savannah, the marshland and the area of the Amazon domain. Data on occurrences of Lutzomyia whitmani were obtained from reports on entomological surveys carried out by the Entomology Group of the National Health Foundation between 1996 and 2000 and by the Entomology Laboratory of the Mato Grosso State Health Department between 2001 and 2006. Entomological surveys were performed in 83 (59.7\%) of the 139 municipalities of Mato Grosso. Lutzomyia whitmani was caught in 70 (84.3\%) municipalities, with widespread distribution in all types of vegetation.
\end{abstract}

Key-words: Lutzomyia whitmani. American tegumentary leishmaniasis. Mato Grosso.

A leishmaniose tegumentar americana (LTA) é uma zoonose de ambientes e de animais silvestres e encontra-se entre as seis doenças infecto-parasitárias de maior importância por representar um dos grandes problemas de saúde pública, devido ao crescente processo de urbanização $0^{59}$. É registrada em todos os estados do Brasil $^{7}$, a região Centro-Oeste ocupa o terceiro lugar dentre as regiões brasileiras em incidência e o primeiro em crescimento da doença ${ }^{13}$. No Estado de Mato Grosso, 100\% dos municípios apresenta registro de casos autóctones ${ }^{6}$ e segundo dados da Vigilância Epidemiológica da Secretaria Estadual de Saúde de Mato Grosso, no período de 2002 a 2006 foram notificados 26.392 casos da doença.

Os insetos implicados na transmissão da LTA em Mato Grosso provêm de ecótopos diversos (Floresta Amazônica, Cerrado e Pantanal). A fauna flebotomínica é variada, sendo assinaladas no estado, espécies relevantes implicadas na transmissão da LTA no Brasil, como Lutzomyia flaviscutellata, Lutzomyia intermedia, Lutzomyia migonei, Lutzomyia umbratilis, Lutzomyia wellcomei e Lutzomyia whitmani ${ }^{18}$.

Lutzomyia whitmani é considerado vetor da Leishmania (Viannia) braziliensis no nordeste, sudeste, centro-oeste e sul do Brasil, sendo também um vetor de Leishmania (Viannia) shawi no norte ${ }^{77}$ e de Leishmania (Viannia) guianenses na Amazônia ${ }^{17}$. É uma espécie de ampla distribuição na América do $\mathrm{Sul}^{23}$ e na região Centro-Oeste, Galati e cols ${ }^{11}$ observaram a antropofilia da espécie, destacando-a como prática exofílica. A espécie apresenta grande frequiência na mata e em galinheiros, sugerindo processo de adaptação ao ambiente antrópico ${ }^{16}$. É oportunista e possui ecletismo alimentar, ajustando seus hábitos à disponibilidade de hospedeiros nos ambientes

1. Laboratório de Entomologia, Secretaria Estadual de Saúde de Mato Grosso, Cuiabá, MT.

Endereço para correspondência: Dra. Nanci Akemi Missawa. Laboratório de Entomologia/SES/MT. Av. Adauto Botelho s/nº, Parque da Saúde, Bairro Coophema, 78085-200 Cuiabá, MT.

Tel: $55653661-2934$

e-mail: nanci.am@terra.com.br

Recebido para publicação em 26/07/2007

Aceito em 07/07/2008 
antrópicos ${ }^{15}$. Segundo Souza e cols ${ }^{21}$, Lutzomyia whitmani pode ser considerada totalmente adaptada a áreas urbanas, sendo capaz de se reproduzir nesses ambientes, e que a LTA pode ser transmitida tanto no peridomicílio quanto no intradomicílio.

0 processo de urbanização da LTA está relacionado a vários fatores como as características ecológicas de cada região, a infectividade das espécies de Leishmanias e os hábitos dos flebotomíneos na transmissão da doença. No Estado de Mato Grosso, Ribeiro e cols ${ }^{19}$ estudaram flebotomíneos de interesse médico na área de influência da Usina de Manso, onde capturaram exemplares de Lutzomyia whitmani. Azevedo e cols ${ }^{3}$ pesquisaram no município de Peixoto de Azevedo, norte do estado, caracterizando 26 espécies de flebotomíneos característicos da região Amazônica, discutindo que a alta densidade de Lutzomyia whitmani poderia estar associada às modificações na cobertura da vegetação original e à plasticidade do vetor às novas condições ambientais, com atividade laboral voltada para a agricultura e mineração.

Rodrigues e cols ${ }^{20}$ realizaram pesquisa entomológica e de domiciliação na região centro-norte e centro-sul de Mato Grosso, onde constataram Lutzomyia whitmani no peridomicílio, em altas densidades. De Luca e cols ${ }^{8}$ estudaram a distribuição de flebótomos em Alta Floresta, região norte do estado, onde detectaram a espécie em remanescentes florestais e em áreas de pastagem.

Missawa e Maciel $^{14}$ listaram 106 espécies de flebotomíneos no Estado de Mato Grosso e observaram que Lutzomyia whitmani ocorreu em áreas de floresta, cerrado e pantanal, assim como em trabalho de Ribeiro e Missawa ${ }^{18}$. A espécie apresentou ampla e uniforme distribuição, registrada em ambiente intra e peridomiciliar, sendo provavelmente a mais adaptada do estado ${ }^{1820}$. Em Mato Grosso do Sul, a ínfima densidade de Lutzomyia whitmani numa área rural do pantanal sul-matogrossense, não indicou risco real de transmissão da LTA naquela área ${ }^{4}$.

0 presente trabalho objetivou verificar a distribuição geográfica do principal vetor da LTA, Lutzomyia whitmani, no Estado de Mato Grosso, visando orientar as ações de prevenção e controle desta endemia.

\section{MATERIAL E MÉTODOS}

O Estado de Mato Grosso (Figura 1) localiza-se na região centro oeste do país, apresenta área geográfica de $903.386,1 \mathrm{~km}^{2}$ e é dividida em 139 municípios e o número de habitantes chega à 2.502.260. Possui três ecossistemas distintos, o cerrado, o pantanal e área de domínio amazônico. Grande parte de seu território é ocupada pela Amazônia Legal, sendo que o extremo sul do estado pertence ao Centro-Sul do Brasil. Tem como limites Amazonas e Pará, ao norte; Tocantins e Goiás, a Leste; Mato Grosso do Sul, ao Sul; Rondônia e Bolívia, a Oeste. Possui extensa rede hidrográfica, abrangendo grande parte das duas maiores bacias do Brasil, a Amazônica e a Platina. 0 clima é tropical quente e subúmido, com chuvas concentradas em janeiro, fevereiro e março e temperatura média anual varia de $24^{\circ} \mathrm{C} \mathrm{a} 42^{\circ} \mathrm{C}$. 0 estado é considerado a maior fronteira agrícola em expansão no Brasil e destaca-se também o turismo, pois a região é muito rica em recursos naturais ${ }^{10}$.

Os dados foram obtidos a partir de relatórios de pesquisa entomológica realizados pelo Núcleo de Entomologia da Fundação Nacional de Saúde (FUNASA) no período entre 1996 e 2000 e entre 2001 a 2006 pelo Laboratório de Entomologia da Gerência de Vigilância de Vetores e Antropozoonoses/Coordenadoria de Vigilância em Saúde Ambiental/Superintendência de Vigilância em Saúde/Secretaria Estadual de Saúde/Mato Grosso (GEVVAN/ COVSAM/SUVSA/SES/MT) em municípios prioritários para a vigilância das leishmanioses. As coletas foram realizadas em levantamentos entomológicos realizados com armadilhas de luz $\mathrm{CDC}^{22}$ e o resultado qualitativo da distribuição espacial foi indicado por presença de Lutzomyia whitmani, ausência de Lutzomyia whitmani ou como município não pesquisado.

\section{RESULTADOS}

No período de estudo, foram realizadas pesquisas entomológicas para flebotomíneos em 83 (59,7\%) dos 139 municípios do Estado de Mato Grosso. 0 principal vetor da LTA, Lutzomyia whitmani, foi capturado em 70 municípios, que corresponde a $84,3 \%$ dos municípios pesquisados, evidenciando uma ampla distribuição da espécie (Figura 2).

A relação entre o tipo de vegetação e a ocorrência de Lutzomyia whitmani evidencia a presença da espécie em todos os tipos de vegetação como áreas de floresta, cerrado e pantanal, incluindo zonas de transição.

\section{DISCUSSÃo}

Espécies de flebotomíneos relacionadas ao processo de urbanização e a transmissão da doença são encontradas em áreas alteradas como plantações de monoculturas, mas também no peridomicílio e no intradomicílio ${ }^{9}$. A expansão da doença para novas fronteiras, provavelmente se deva ao desmatamento das áreas situadas entre as grandes cidades, em função de implantação de diversos projetos de desenvolvimento ${ }^{612}$, enviesados por uma extensa rede de rodovias de acesso.

Costa e cols ${ }^{7}$ destacaram que Lutzomyia whitmani pode ser encontrado nas cinco regiões do Brasil, presentes em grande número de municípios dos Estados de Roraima, Acre, Tocantins e Mato Grosso do Sul, em associação com uma variedade de tipos de vegetação como florestas, cerrado e caatinga.

Entre as pesquisas entomológicas realizadas no Estado de Mato Grosso, Aguiar e Medeiros ${ }^{2}$ apresentaram várias listas de flebotomíneos entre as quais, Lutzomyia whitmani foi considerada em processo de domiciliação e de ampla distribuição, particularmente pela sua adaptação a habitats menos especializados ou mais diversificados.

0 conhecimento da ocorrência da LTA em todos os municípios do estado e a ampla distribuição de Lutzomyia whitmani sugere 


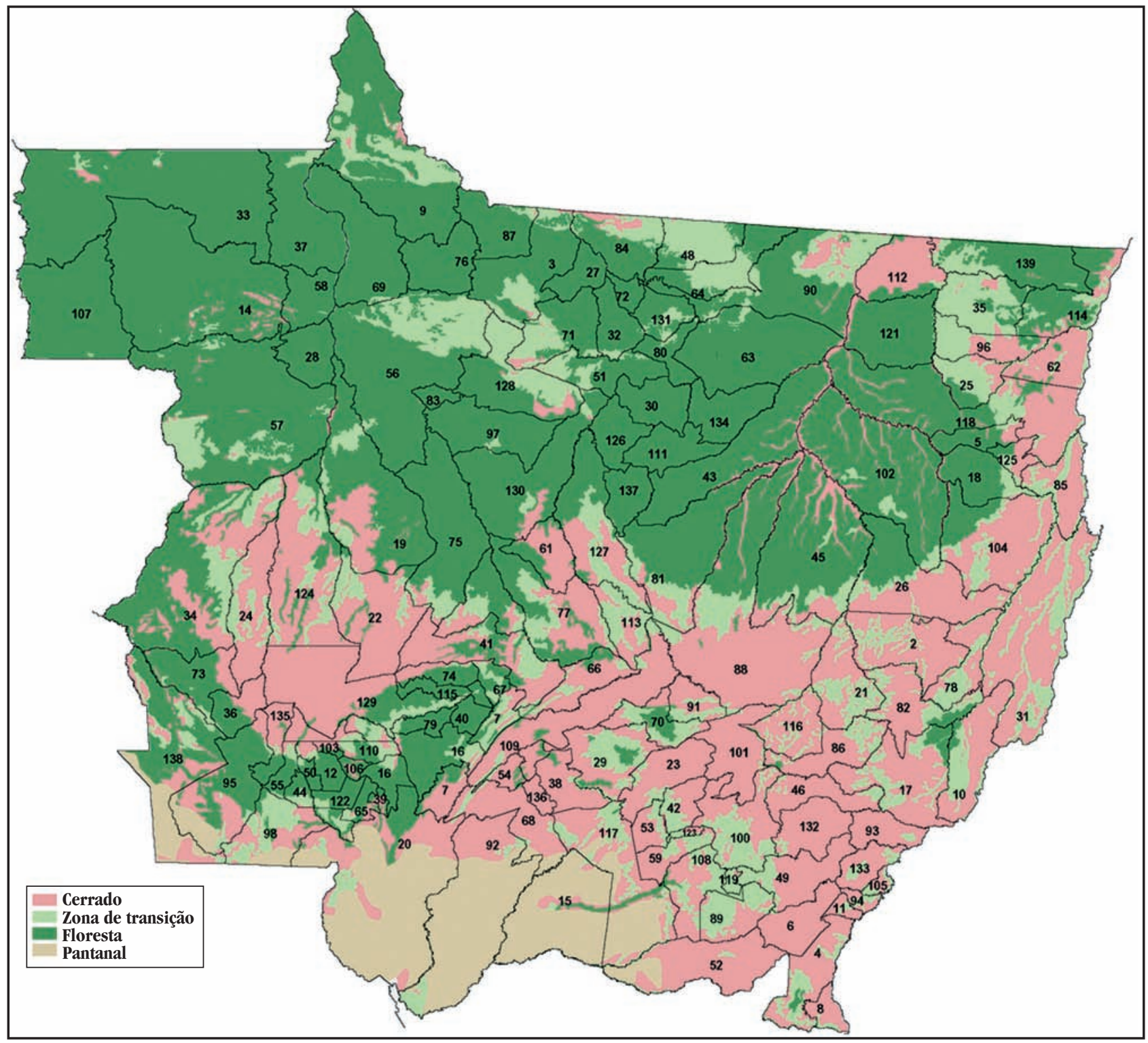

\begin{tabular}{|c|c|c|c|c|c|}
\hline \multicolumn{3}{|l|}{ Relação dos } & \multirow[b]{2}{*}{$\begin{array}{l}\text { 74. Nova Marilândia } \\
\text { 75. Nova Maringá } \\
\text { 76. Nova Monte Verde } \\
\text { 77. Nova Mutum } \\
\text { 78. Nova Nazaré } \\
\text { 79. Nova Olímpia } \\
\text { 80. Nova Santa Helena } \\
\text { 81. Nova Ubiratã } \\
\text { 82. Nova Xavantina } \\
\text { 83. Novo Horizonte do Norte } \\
\text { 84. Novo Mundo } \\
\text { 85. Novo Santo Antônio } \\
\text { 86. Novo São Joaquim } \\
\text { 87. Paranaita } \\
\text { 88. Paranatinga } \\
\text { 89. Pedra Preta } \\
\text { 90. Peixoto de Azevedo } \\
\text { 91. Planalto da Serra } \\
\text { 92. Poconé } \\
\text { 93. Pontal do Araguaia } \\
\text { 94. Ponte Branca } \\
\text { 95. Pontes e Lacerda } \\
\text { 96. Porto Alegre do Norte } \\
\text { 97. Porto dos Gaúchos } \\
\text { 98. Porto Esperidião } \\
\text { 99. Porto Estrela } \\
\text { 100. Poxoréo }\end{array}$} & \multirow[b]{2}{*}{\begin{tabular}{|l|} 
101. Primavera do Leste \\
102. Querência \\
103. Reserva do Cabaçal \\
104. Ribeirão Cascalheira \\
105. Ribeirãozinho \\
106. Rio Branco \\
107. Rondolândia \\
108. Rondonópolis \\
109. Rosário Oeste \\
110. Salto do Céu \\
111. Santa Carmem \\
112. Santa Cruz do Xingú \\
113. Santa Rita do Trivelato \\
114. Santa Terezinha \\
115. Santo Afonso \\
116. Santo Antonio do Leste \\
117. Santo Antonio do Leverger \\
118. São Félix do Xingú \\
119. São José do Povo \\
120. São José do Rio Claro \\
121. São José do Xingú \\
122. Sãa José dos Quatro Marcos \\
123. São Pedro da Cipa \\
124. Sapezal \\
125. Serra Nova Dourada \\
126. Sinop
\end{tabular}} & \multirow[b]{2}{*}{$\begin{array}{l}\text { 127. Sorriso } \\
\text { 128. Tabaporã } \\
\text { 129. Tangará da Serra } \\
\text { 130. Tapurah } \\
\text { 131. Terra Nova do Norte } \\
\text { 132. Tesouro } \\
\text { 133. Torixoréu } \\
\text { 134. União do Sul } \\
\text { 135. Vale de São Domingos } \\
\text { 136. Várzea Grande } \\
\text { 137. Vera } \\
\text { 138. Vila Bela de Santíssima } \\
\text { Trindade } \\
\text { 139. Vila Rica }\end{array}$} \\
\hline $\begin{array}{l}\text { al } \\
\text { 3oa } \\
\text { oresta } \\
\text { caguaia } \\
\text { oa Vista } \\
\text { arças } \\
\text { araguai } \\
\text { qquari } \\
\text { is } \\
\text { laiana } \\
\text { lainha } \\
\text { utanga } \\
\text { ápolis }\end{array}$ & Norte & a & & & \\
\hline
\end{tabular}

Figura 1 - Área de estudo: Estado de Mato Grosso: mapa de vegetação. 


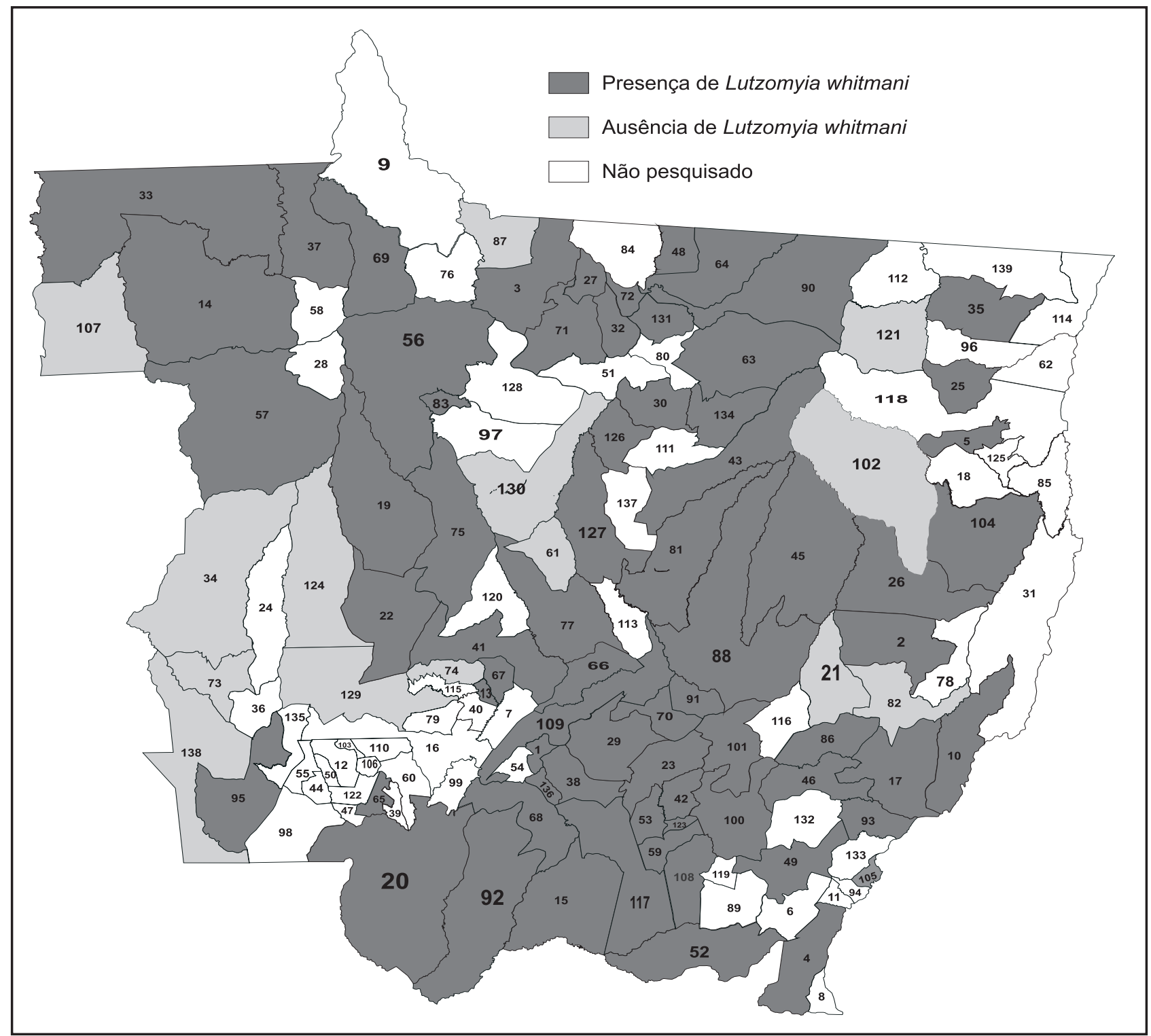

\begin{tabular}{|c|c|c|c|c|c|}
\hline \multicolumn{3}{|c|}{ Relação dos Municípios de Mato Grosso } & \multirow[b]{2}{*}{$\begin{array}{l}\text { 74. Nova Marilândia } \\
\text { 75. Nova Maringá } \\
\text { 76. Nova Monte Verde } \\
\text { 77. Nova Mutum } \\
\text { 78. Nova Nazaré } \\
\text { 79. Nova Olímpia } \\
\text { 80. Nova Santa Helena } \\
\text { 81. Nova Ubiratã } \\
\text { 82. Nova Xavantina } \\
\text { 83. Novo Horizonte do Norte } \\
\text { 84. Novo Mundo } \\
\text { 85. Novo Santo Antônio } \\
\text { 86. Novo São Joaquim } \\
\text { 87. Paranaita } \\
\text { 88. Paranatinga } \\
\text { 89. Pedra Preta } \\
\text { 90. Peixoto de Azevedo } \\
\text { 91. Planalto da Serra } \\
\text { 92. Poconé } \\
\text { 93. Pontal do Araguaia } \\
\text { 94. Ponte Branca } \\
\text { 95. Pontes e Lacerda } \\
\text { 96. Porto Alegre do Norte } \\
\text { 97. Porto dos Gaúchos } \\
\text { 98. Porto Esperidião } \\
\text { 99. Porto Estrela } \\
\text { 100. Poxoréo }\end{array}$} & \multirow[b]{2}{*}{$\begin{array}{l}\text { 101. Primavera do Leste } \\
\text { 102. Querência } \\
\text { 103. Reserva do Cabaçal } \\
\text { 104. Ribeirão Cascalheira } \\
\text { 105. Ribeirãozinho } \\
\text { 106. Rio Branco } \\
\text { 107. Rondolândia } \\
\text { 108. Rondonópolis } \\
\text { 109. Rosário Oeste } \\
\text { 110. Salto do Céu } \\
\text { 111. Santa Carmem } \\
\text { 112. Santa Cruz do Xingú } \\
\text { 113. Santa Rita do Trivelato } \\
\text { 114. Santa Terezinha } \\
\text { 115. Santo Afonso } \\
\text { 116. Santo Antonio do Leste } \\
\text { 117. Santo Antonio do Leverger } \\
\text { 118. São Félix do Xingú } \\
\text { 119. São José do Povo } \\
\text { 120. São José do Rio Claro } \\
\text { 121. São José do Xingú } \\
\text { 122. São José dos Quatro Marcos } \\
\text { 123. São Pedro da Cipa } \\
\text { 124. Sapezal } \\
\text { 125. Serra Nova Dourada } \\
\text { 126. Sinop }\end{array}$} & \multirow[b]{2}{*}{$\begin{array}{l}\text { 127. Sorriso } \\
\text { 128. Tabaporã } \\
\text { 129. Tangará da Serra } \\
\text { 130. Tapurah } \\
\text { 131. Terra Nova do Norte } \\
\text { 132. Tesouro } \\
\text { 133. Torixoréu } \\
\text { 134. União do Sul } \\
\text { 135. Vale de São Domingos } \\
\text { 136. Várzea Grande } \\
\text { 137. Vera } \\
\text { 138. Vila Bela de Santíssima } \\
\text { Trindade } \\
\text { 139. Vila Rica }\end{array}$} \\
\hline $\begin{array}{l}\text { Acorizal } \\
\text { Água Boa } \\
\text { Alta Floresta } \\
\text { llto Araguaia } \\
\text { Alto Boa Vista } \\
\text { Alto Garças } \\
\text { llto Paraguai } \\
\text { Ulto Taquari } \\
\text { Apiacás } \\
\text { Araguaiana } \\
\text { Araguainha } \\
\text { Araputanga } \\
\text { Arenápolis } \\
\text { Aripuanã } \\
\text { Barão de Melgaço } \\
\text { Barra do Bugres } \\
\text { Barra do Garças } \\
\text { Bom Jesus do Araguaia } \\
\text { Brasnorte } \\
\text { Cáceres } \\
\text { Campinápolis } \\
\text { Campo Novo do Parecis } \\
\text { Campo Verde } \\
\text { Campos de Júlio }\end{array}$ & $\begin{array}{l}\text { 25. Canabrava do Norte } \\
\text { 26. Canarana } \\
\text { 27. Carlinda } \\
\text { 28. Castanheira } \\
\text { 29. Chapada dos Guimarães } \\
\text { 30. Claúdia } \\
\text { 31. Cocalinho } \\
\text { 32. Colider } \\
\text { 33. Colniza } \\
\text { 34. Comodoro } \\
\text { 35. Confresa } \\
\text { 36. Conquista D'oeste } \\
\text { 37. Cotriguaçu } \\
\text { 38. Cuiabá } \\
\text { 39. Curvelândia } \\
\text { 40. Denise } \\
\text { 41. Diamantino } \\
\text { 42. Dom Aquino } \\
\text { 43. Feliz Natal } \\
\text { 44. Fioueirópolis D' Oeste }\end{array}$ & a & & & \\
\hline
\end{tabular}

Figura 2 - Distribuição geográfica de Lutzomyia whitmani no Estado de Mato Grosso. 
que esta espécie possa estar envolvida no ciclo de transmissão da Leishmania (Viannia) braziliensis, visto que pesquisa com sondas de DNA, realizada por Andrade e cols ${ }^{1}$ demonstraram que o complexo Leishmania braziliensis é o grupo predominante infectando pacientes humanos no Estado de Mato Grosso.

A distribuição de Lutzomyia whitmani nos diferentes ecótopos de Mato Grosso reflete o entendimento sobre a cadeia de transmissão e seus diferentes perfis eco-epidemiológicos, importantes para direcionar as ações de controle da doença mais adequadas à situação local.

\section{AGRADECIMENTOS}

Aos técnicos do Laboratório de Entomologia da Secretaria Estadual de Saúde de Mato Grosso pelos trabalhos de campo e de laboratório, e também pela amizade e agradável convivência.

\section{REFERÊNCIAS}

1. Andrade ASR, Fernandes O, Hueb M, Carvalho MLR, Fontes CJ, Melo MN. The use of radionuclide DNA probe technology for epidemiological studies of tegumentary leishmaniasis in Mato Grosso State. Brazilian Archives of Biology and Technology 48:201-204, 2005.

2. Aguiar GM, Medeiros WM. Distribuição e Habitats. In: Rangel EF, Lainson R (eds) Flebotomíneos do Brasil, Editora Fundação Oswaldo Cruz, Rio de Janeiro, p. 207-255, 2003.

3. Azevedo ACR, Souza NA, Meneses CRV, Costa WA, Lima JB, Rangel EF. Ecology of sand flies (Diptera: Psychodidae: Phlebotominae) in the north of State of Mato Grosso, Brazil. Memórias do Instituto Oswaldo Cruz 97:459-464, 2002.

4. Braga-Miranda LC, Miranda M, Galati EAB. Phlebotomine fauna in a rural area of the Brazilian Pantanal. Revista de Saúde Pública 40:324-326, 2006.

5. Camargo LB, Langoni H. Impact of leishmaniasis on public health. Journal of Venomous Animals and Toxins including Tropical Diseases 12:527-548, 2006.

6. Costa JML. Epidemiologia das leishmanioses no Brasil. Gazeta Médica da Bahia 75:3-17, 2005.

7. Costa SM, Cechinel M, Bandeira V, Zannuncio JC, Lainson R, Rangel EF. Lutzomyia (Nyssomyia) whitmani s.l. (Antunes \& Coutinho, 1939) (Diptera: Psychodidae: Phlebotominae): geographical distribution and the epidemiology of American cutaneous leishmaniasis in Brazil - Mini review. Memórias do Instituto Oswaldo Cruz 102:149-153, 2007.

8. De Luca AS, Vasconcelos HL, Barrett TV. Distribution of sandflies (Diptera: Phlebotominae) in forest remnants and adjacent matrix habitats in Brazilian Amazonian. Brazilian Journal of Biology 63:401-410, 2003.
9. Dias ES, França-Silva JC, Silva JC, Monteiro EM, Paula KM, Gonçalves CM, Barata RA. Flebotomíneos (Diptera: Psychodidae) de um foco de leishmaniose tegumentar do estado de Minas Gerais. Revista da Sociedade Brasileira de Medicina Tropical 40:49-52, 2007.

10. Ferreira JCV. Mato Grosso e seus municípios. Editora Buriti. Cuiabá/MT, 2001.

11. Galati EAB, Nunes VLB, Dorval MEC, Oshiro ET, Cristaldo E, Espíndola MA, Rocha HC, Garcia WB. Estudo dos flebotomíneos (Diptera, Psychodidae), em área de leishmaniose tegumentar, no estado de Mato Grosso do Sul, Brasil. Revista de Saúde Pública 30:115-128, 1996.

12. Leonardo FS, Rebêlo JMM. A periurbanização de Lutzomyia whitmani em área de foco de leishmaniose cutânea, no Estado de Maranhão, Brasil. Revista da Sociedade Brasileira de Medicina Tropical 37:282-284, 2004.

13. Ministério da Saúde. Fundação Nacional de Saúde. Manual de vigilância da leishmaniose tegumentar americana. Brasília, 2007.

14. Missawa NA, Maciel GBML. List of species in the genus Lutzomyia, França, 1924 (Psychodidae, Phlebotominae) from the state of Mato Grosso. Revista da Sociedade Brasileira de Medicina Tropical 40:11-14, 2007.

15. Muniz LHG, Rossi RM, Nietzke HC, Monteiro WM, Teodoro U. Estudo dos hábitos alimentares de flebotomíneos em área rural no sul do Brasil. Revista de Saúde Pública 19:1087-1093, 2006.

16. Oliveira AG, Andrade $\mathrm{F}^{0} \mathrm{JD}$, Falcão AL, Brazil RP. Estudo dos flebotomíneos (Diptera, Psychodidae, Phlebotominae) na zona urbana da cidade de Campo Grande, Mato Grosso do Sul, Brasil, 1999-2000. Cadernos de Saúde Pública 19:933-944, 2003.

17. Rangel EF, Lainson R. Ecologia das leishmanioses. In: Rangel EF, Lainson $\mathrm{R}$ (eds) Flebotomíneos do Brasil, Editora Fundação Oswaldo Cruz, Rio de Janeiro, p.291-309, 2003.

18. Ribeiro ALM, Missawa NA. Spatial distribution of phlebotomine species in the state of Mato Grosso, Brazil, in the period of 1996 to 2001. Entomología y Vectores 9:33-34, 2002.

19. Ribeiro ALM, Oliveira RC, Miyazaki RD, Pignat WA. Inventário dos vetores da leishmaniose (Diptera: Psychodidae: Phlebotominae) em Área de Aproveitamento Múltiplo de Manso, Chapada dos Guimarães, Nobres, Rosário Oeste e Nova Brasilândia/Mato Grosso/Brasil (Dados preliminares). Revista da Sociedade Brasileira de Medicina Tropical 35:313, 2002.

20. Rodrigues H, Missawa NA, Costa LB, Lima GBM, Ramos SR, Moraes Filho VC, Souza CO, Ribeiro ALM, Silva AM, Barros IM. A interferência humana como fator de agravamento ambiental e a domiciliação dos vetores da leishmaniose tegumentar americana no estado de Mato Grosso, no período de 2001 e 2002. Revista da Abrasco 8: 414, 2003.

21. Souza CM, Pessanha JE, Barata RA, Monteiro EM, Costa DC, Dias ES. Study on phlebotomine sand fly (Diptera: Psychodidae) fauna in Belo Horizonte, State of Minas Gerais, Brazil. Memórias do Instituto Oswaldo Cruz 99:795-803, 2004.

22. Sudia WA, Chamberlain RW. Battery-operated light trap: an improved model. Mosquitoes News 22:126-129, 1962.

23. Young DG, Duncan MA. Guide to the identification and geographic distribution of Lutzomyia sand files in México, the West Indies, Central and South America (Diptera: Psychodidae). Associated Publishers American Entomological Institute. Florida, USA, 1994. 